\title{
Probabilistic evaluation of renewable energy curtailment under forward electricity market
}

\author{
Jiheng Jiang ${ }^{1}$, Ying Qiao ${ }^{1}$, Zongxiang $\mathrm{Lu}^{1}$, Liang $\mathrm{Ran}^{2}$, Ming $\mathrm{Ma}^{2}$, Jin $\mathrm{Li}^{3}$, Feng Wang ${ }^{2}$ \\ ${ }^{1}$ State Key Lab of Control and Simulation of Power Systems and Generation Equipments, Dept. of Electrical Engineering, Tsinghua \\ University, Beijing 100084, China \\ ${ }^{2}$ State Grid Gansu Provincial Electric Power Company, Lanzhou, 730000, China \\ ${ }^{3}$ Key Laboratory of Wind Power Integration Operation and Control of Gansu Province, Lanzhou 730070, China
}

\begin{abstract}
Forward electricity market has emerged as a pivotal section for the electricity transaction to keep stakeholders away from price risk and electricity shortage. But the forward contract of conventional unit partially limits the unit output before operation, leading to the curtailed generation share of renewable energy source (RES). Modeling and assessing this impact is of great significance for system planning and market supervision. The central thesis of this paper is to find out the impact of forward market on RES curtailment. A probabilistic evaluation model for RES accommodation is proposed, taking the curtailment rate as a main evaluation index. We mainly research financial contract and physical contract, modeling the impact of them on thermal unit minimum load capacity and power load in evaluation model. The simulation is conducted in a simplified system, which reveals the change of RES curtailment with renege penalty, contract price and execution generation curve.
\end{abstract}

\section{Introduction}

The forward market is an important part of the power market, acting as a complement for the spot market. The forward market refers to the market in which forward contracts are traded, and transactions are settled on a certain date in the future. The content of forward contract is usually the stakeholders agree to buy or sell a certain quantity of electricity at a certain price in the future [1]. So forward market plays a vital role in avoiding the price risk of electricity and detecting the demand in advance and was established in many countries.

Forward market is a classic market form in Chinese power system. For example, annual electricity plan and load entity direct transaction are the typical forward transaction without full marketization. With electricity marketization in China, forward market has become a central issue. In some provinces, the contract between thermal unit and load entity increase the lower bound of conventional unit forced power, leading to reduced generation share of RES. A primary concern of forward market here is how to evaluate the effect of precontracted electricity on future RES curtailment in a relatively long term, such as a month or a season. This issue is of great significance within the field of future power market construction.

Previous research was mainly focused on the market situation and economic analysis of forward market. To date, several studies have investigated the transaction mode of forward market in power market reform starting at 2015 in China [2], [3]. Reference [4] builds a new dynamic model to evaluate the effect of forward market on cyclical price behavior in electricity markets. An equilibrium model for joint forward contracts and dayahead markets are proposed in [5], and the complicated market behavior of producers and consumers are also modeled. Agent-based model is a fundamental path to model the price building, transaction and operation of the forward market, which is used in [6]. In this study, authors consider renewable energy situation as boundary conditions but not dependent variable. Similarly, research [7] considers renewable energy volatility as an independent variable and indicates RES may undermine the role of forward contract, decreasing incentive of power plants. Another hot topic is the coordination of unit commitment and forward contract, on which reference [8] and [9] propose multi-time-range model for daily operation and long-term trading plans. Reference [10] analyzes the forward contract of interruptible load and gives an optimal management method, including purchase process and dispatch rule. The effect of forward market are only the auxiliary work of the above papers. What is not yet clear is the constrain of thermal unit minimum generation share caused by forward market and impact of forward market on RES curtailment in a long time scale rather than sequential analysis.

This paper attempts to explore this issue by building a probabilistic model based on probabilistic production simulation (PPS) method to assess RES accommodation. The two types of forward market considered are financial contract and physical contract. This paper begins by the probabilistic evaluation model for RES curtailment in

\footnotetext{
* Corresponding author: jih17@mails.tsinghua.edu.cn
} 
section 2. It will then go on to the model of financial contract and physical contract in the perspective of probability, described in section 3 . Section 4 presents the result of numerical experiment that shows the change of RES curtailment with renege penalty, contract price of financial contract and execution curve shape, contract electricity of physical contract.

\section{Basic principle of evaluation model}

\subsection{Curtailment power evaluation model}

The main step to assess the impact of forward contract on renewable energy generation is building the evaluation model of renewable energy accommodation. Based on PPS, we illustrate the basic principle of generation curtailment and accommodation evaluation from a probabilistic perspective. Assuming that the sequential power load curve could be collected from TSO and be relatively stable in probability characteristic, which makes it possible to predict the future load curve on the basis of historical curve multiplied by rate. According to sequential load curve, the equivalent load duration curve (ELDC) $F_{L}(x)$ is defined as

$$
F_{L}(X)=\int_{X}^{P_{\text {Lmax }}} f_{L}(x) d x
$$

where $x$ is value of power load or capacity of generation. $P_{\mathrm{L} \text { max }}$ is the maximum load, MW. $f_{\mathrm{L}}(x)$ is the probability distribution function (PDF) of power load, calculated from historical data.

Power balance in PPS is the process that power sources fill the area between ELDC and X-axis in a specific order, shown in Fig. 1. Physically, this area is the total energy needed, or the accommodation area for sources. In Fig. 1, power source A, B, D and E are arranged in different positions under ELDC, where the width of each position is the capacity of generation. Source A,B and E are single state units, which means there is only one forced outage rate (FOR) in whole capacity, while source D have three different FOR in the variable states. If ELDC is less than 1, the generation arranged in that positon may face with curtailing energy, represented by yellow part in Fig. 1. Curtailment energy $E_{\text {cur }}$ depends on the yellow part and FOR of corresponding units, which can be stated as

$$
E_{\text {cur }}=T \cdot \sum_{I}\left(1-q_{i}\right) \cdot \int_{x_{i}}^{x_{i}+C_{i}}\left(1-F_{L}(x)\right) d x
$$

where $I$ is the state set of a unit, and $T$ is the calculation time range, usually a month. $q_{i}$ is the FOR of state $i$. $C_{i}$ is the capacity of state $i . x_{i}$ is the starting point in the $\mathrm{X}$-axis of state $i$. is

Correspondingly, accommodation energy of this unit

$$
E_{a c c}=T \cdot \sum_{I}\left(1-q_{i}\right) \cdot \int_{x_{i}}^{x_{i}+C_{i}} F_{L}(x) d x
$$

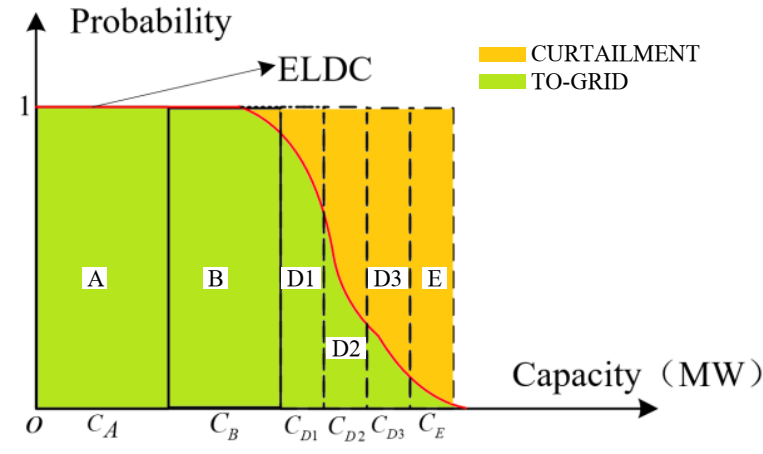

Fig. 1 Evaluation principle of generation curtailment and accommodation

It is obvious that the energy calculated by (2) and (3) are expectation value in a period. The process are independent from the sequential data, simplifying load prediction. As for unit types, totally controllable units, including conventional thermal unit, nuclear unit, are usually modeled as one-state or two-state unit which consists of forced capacity and regulation capacity. Curtailment energy is surplus generation capability but no fuel waste for them. So we usually focus on the curtailment energy of RES. Wind power and PV are usually types of multi-state, because they are constrained by natural resource. In that case, the capacity-probability pairs set of renewable energy can be calculated from PDF with discretization, shown in Fig. 2.

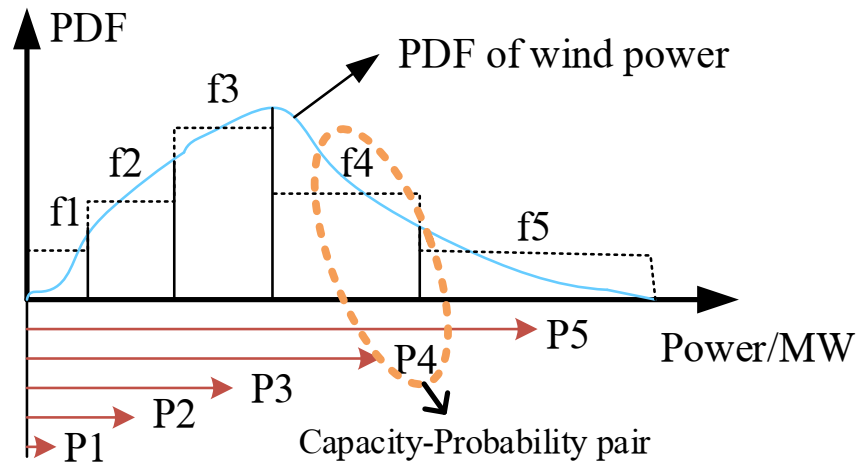

Fig. 2 Discretized capacity-probability pairs of wind power

\subsection{Interaction and arrangement of power sources}

With reference to the dispatching principle, the base load, especially part A and part B in Fig. 1, is supported by thermal units and nuclear units. And renewable energy is regarded as "middle power", supporting the shoulder load that is represented by part D in Fig. 1 for RES can't provide regulation service to track load. In PPS, the wind power will be filled after the minimum load capacity of the non-heating thermal power are involved. Sometimes, hydropower station with insufficient reservoir is arranged in the same position with wind power. However, this paper is concentrated on the fluctuant RES, mainly the wind power, without detailed research on hydropower. After that, the load not supported is peak load that is the 
main generation area for thermal unit regulation capacity.

The existing studies show that a large number of conventional units with too much minimum load will cause severe curtailment, which indicates the base load part, such as part A and part B in Fig. 1, occupies energy area of RES. The amount of power consumption shown in Part D of Fig. 1 will decrease as the thermal unit capacity increases. The closer to peak load the arrangement position is, the more possibility to curtail power the unit has. Therefore, the PPS method has a principle advantage in the evaluation of medium and long-term.

Describing the interaction of conventional power and RES, we still need to research how FOR influence the ELDC. If FOR of unit A is less than 1, it indicates this unit may black out randomly, adding power load to other units arranged behind A equivalently. So ELDC must be modified after a unit is arranged to support load. The modification formulation is

$$
F^{(i)}(x)=\sum q_{i} F^{(i-1)}\left(x-C_{i}+\mathrm{C}_{E}\right),(i=1,2,3,4 \cdots)
$$

where $F^{(i)}(x)$ and $F^{(i-1)}(x)$ is the new ELDC and former one. $C_{i}$ is the available capacity of state $i . \mathrm{C}_{E}$ is the rate capacity of arranged unit.

The ELDC will become more flat and higher, increasing generation area for next unit, for there is almost no unit with zero-FOR. In particular, this tendency is the most distinct after modified by RES.

\section{Probabilistic model of forward market}

\subsection{Financial contract}

For the purpose of financial contract in forward market, the design is only for avoiding the risk of energy price fluctuation rather than energy production uncertainty. Existing research has an assumption that the financial contract has no impact on real-time dispatch result. In that case, RES generation area will not be reduced by these virtual contract. In other word, financial contract may be decoupled from physical production. The previous model sounds reasonable but not practical. The unbalance penalty after contract period is becoming higher and price gap between contract and real time caused by congestion is highly fluctuant. We can conclude that high accounting penalty could form invisible constraints on physical execution.

To model this constrain effect, we assume the penalty would increase the minimum load capacity of a thermal unit bound with a load entity by forward contract. Part A in Fig. 1 may be broadened, if unit A is in a contract and the penalty is high. The modified minimum load capacity $C_{\text {min }}$ is defined as follows:

$$
C_{\min }= \begin{cases}C_{\min }^{o r i}, & \left(\frac{Q_{f}}{T} \leq C_{\min }^{o r i}\right) \\ C_{\min }^{o r i}+\eta \cdot\left(\frac{Q_{f}}{T}-C_{\min }^{o r i}\right), & \left(\frac{Q_{f}}{T}>C_{\min }^{o r i}\right)\end{cases}
$$

where $C_{\mathrm{min}}^{\text {ori }}$ is the physical minimum capacity of unit.
$Q_{f}$ is the contract energy in period $T . \eta$ is the modification coefficient for $C_{\min }$. Then, $\eta$ is proportional to penalty price and inversely proportional to contract price, which is stated as

$$
\eta=\frac{2}{1+e^{-\left(\frac{m_{p}}{m_{e}} \cdot \frac{m_{e s}}{m_{p s}}\right.}}-1
$$

where $m_{p}$ is the average penalty price for unexecuted energy per MWh. And $m_{e}$ is the average price of contract energy per MWh. $m_{p s}, m_{e s}$ that are pre-determined before PPS are the reference value of $m_{p}$ and $m_{e}$.

In addition, the model proposed above links market price to system physical operation and implies that the minimum load capacity of thermal unit may increase at any time. That's why the minimum load capacity part under ELDC is still a rectangle.

\subsection{Physical contract}

The section above introduces a power source modification model for financial contract. As for physical contract, this section will propose an ELDC modification model. Physical contract between power plant and load entity contains detailed contract time, power and price, which can be seen as a deterministic and fixed daily load curve. The following analysis uses unit $\mathrm{A}$ as an example to illustrate the modeling method.

The time when physical contract of unit A is operated in a day is $\left[0, t_{s}\right] \cup\left[t_{e}, 24\right]$. We just research units only with a physical contract but no financial contract. So the intraday load sequence $L(t)$ is separated into two series:

$$
\begin{aligned}
L_{1}(t) & = \begin{cases}0, & t \in\left[0, t_{s}\right] \cup\left[t_{e}, 24\right] \\
L(t), & t \in\left[t_{s}, t_{e}\right]\end{cases} \\
L_{2}(t) & = \begin{cases}L(t), & t \in\left[0, t_{s}\right] \cup\left[t_{e}, 24\right] \\
0, & t \in\left[t_{s}, t_{e}\right]\end{cases} \\
F_{L 1}(x), F_{L 2}(x) \text { are the ELDC of } L_{1}(t), L_{2}(t) &
\end{aligned}
$$
And $f_{L 1}(x), f_{L 2}(x)$ are PDF (refer to formula(1)). Physical contract works on $L_{2}(t)$ and reduces the accommodation area for RES. So $F_{L 2}(x)$ and $f_{L 2}(x)$ must be modified before the calculation of PPS. We define $L_{2}^{\text {new }}(t)$ is

$$
L_{2}^{\text {new }}(t)=L_{2}(t)-L_{p}(t)
$$

where $L_{p}(t)$ is the equivalent power curve of physical contract applied in PPS. It eliminates the minimum capacity because only the contract load part overtop the minimum load capacity will decrease the area for RES.

$$
L_{p}(t)= \begin{cases}L_{p}^{\text {ori }}(t)-C_{\min }^{\text {ori }}, & L_{p}^{\text {ori }}(t) \geq C_{\mathrm{min}}^{\text {ori }} \\ 0, & L_{p}(t)<C_{\mathrm{min}}^{\text {ori }}\end{cases}
$$

where $L_{p}^{\text {ori }}(t)$ is actual contract load curve.

A major assumption is the power load is regular in long term, which means the power level and PDF of intraday load in $\left[0, t_{s}\right] \cup\left[t_{e}, 24\right]$ is almost the same in a month. And the contract power is totally fixed in the 
same time everyday. The $\left(L_{2}, L_{P}\right)$ is joint random variable of load and contract power. And the PDF of this variable is defined as $f_{L}\left(l_{2}, l_{p}\right)$. The $\operatorname{PDF}$ of $L_{2}^{\text {new }}(t)$ is defined as $f_{L}\left(l_{2}^{\text {new }}\right)$ calculated by following convolution formula.

$$
f_{L}\left(l_{2}^{\text {new }}\right)=\int\left[f_{L . l_{2}}\left(l_{2}^{\text {new }}+l_{p}\right) \cdot f_{L . l_{p}}\left(l_{p}\right)\right] \mathrm{d} l_{p}
$$

where $f_{L . l_{2}}($.$) is the edge distribution of l_{2}$ for PDF and $f_{L . l_{p}}\left(\right.$.) the edge distribution of $l_{p}$.

Then, $F_{L 2}(x)$ is corrected to $F_{L 2}^{\text {new }}(x)$ which is the integral of $f_{L}\left(l_{2}^{n e w}\right)$. And the modified ELDC $F_{L}^{\text {new }}(x)=F_{L 1}(x)+F_{L 2}(x)$ is generated. In practice, $f_{L}\left(l_{2}, l_{p}\right)$ can be calculated through statistics. Two edge distribution functions are easy to get from $f_{L}\left(l_{2}, l_{p}\right)$. Even though the load data is discrete rather than continuous, the principle of integral is still applicative.

\subsection{Process of forward contract effect evaluation}

The process of PPS for evaluation calculation is stated as following.

Step 1. Generate the ELDC based on the historical data of generation load in the region.
Step 2. For all conventional thermal units, two sections of every unit are separated in accordance with the minimum power and adjustable power.

Step 3. Calculate the equivalent minimum load capacity of thermal units that are in financial contract through (5). Arrange the minimum power part of the conventional thermal units to support the basic load.

Step 4. Modify the ELDC according to the fixed load curve in physical contract through formula (7)-(10).

Step 5. Arrange wind power into production.

Step 6. Calculate the abandoned wind power energy $E_{w c}$ through formula (2).

Step 7. Filled the rest area with the adjustable power output of conventional thermal units.

\section{Simulation and results}

\subsection{Data set}

A simplified simulation system, consisting of three thermal power units, an equivalent centralized wind farm and several load entities, was used to analyze the effect of forward market. The total capacity of power source is 2050MW. Table 1 shows details of power source and system generation load.

Table 1 Parameters of power source and load in system

\begin{tabular}{|c|c|c|c|c|c|c|c|c|}
\hline \multicolumn{3}{|c|}{ Thermal } & \multicolumn{3}{|c|}{ Wind power } & \multicolumn{3}{|l|}{ Load } \\
\hline $\begin{array}{l}\text { Unit } \\
\text { No. }\end{array}$ & Capacity(MW) & $\begin{array}{l}\text { Min load } \\
(\mathrm{MW})\end{array}$ & $\operatorname{Max}(\mathrm{MW})$ & $\operatorname{Min}(\mathrm{MW})$ & Average(MW) & $\operatorname{Max}(\mathrm{MW})$ & Min(MW) & Average(MW) \\
\hline 1 & 600 & 240 & 596.8 & 122.7 & 481.2 & 1203.8 & 737.6 & 985.7 \\
\hline 2 & 300 & 120 & & & & & & \\
\hline 3 & 300 & 120 & Rated capa & ty: $850 \mathrm{MW}$ & & Total energ & :709.7GWh & \\
\hline
\end{tabular}

Sequential load curve and wind power curve of 15minute time precision are proportionately modified from the data of typical province power system in northwestern China. The simulation is conducted in one month. Fig. 3 (a) shows the typical curve of load and wind power supported by thermal unit minimum load capacity in first 5 days. Fig. 3 (b) shows the PDF of wind power. The result reveals that there has been a slight RES curtailment with all thermal units on. The curtailment energy of whole month is $22852.6 \mathrm{MWh}$ and curtailment rate is $6.05 \%$.

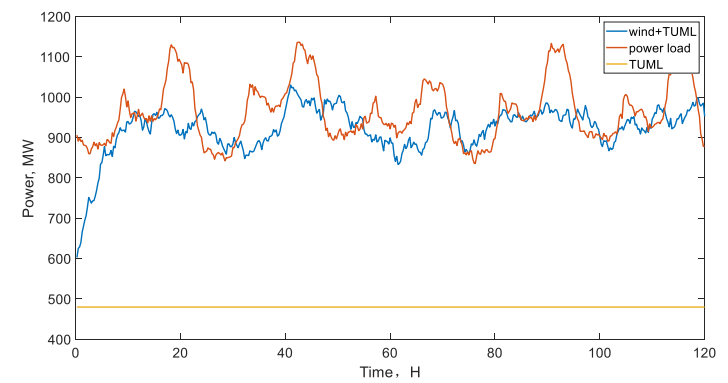

(a) Typical curve of load and wind power (TUML-Thermal unit minimum load)

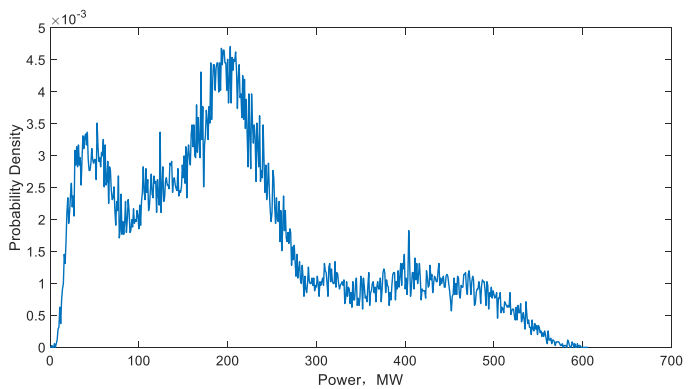

(b)PDF of wind power

Fig. 3 Sequential curve and statistics of power source

\subsection{Effect of financial contract}

In this numerical trial, we assume only thermal unit No.2 has a financial contract with other load entities but no physical contract. Fig. 4 shows the value change of $\eta$ with variation of penalty and contract price according to formula(6). The reference value of contract price and renege penalty are 1 and 10 yuan/KWh, respectively. The figure can show that the increase of penalty and decrease of contract price will make coefficient climb up. 


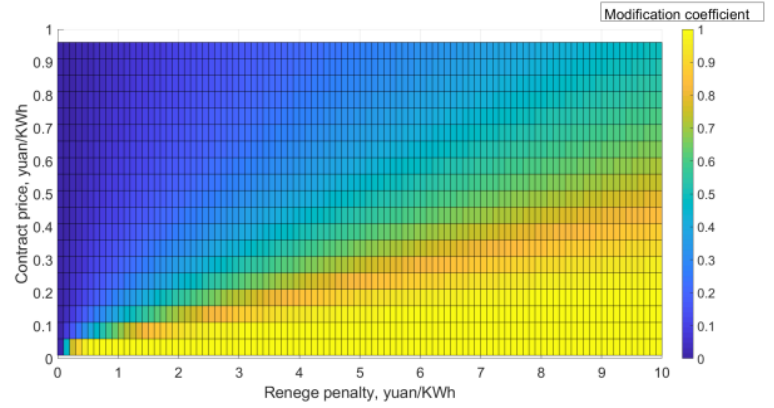

Fig. 4 Trends of modification coefficient $\eta_{\text {with renege penalty }}$ and contract price.

Fig. 5 shows the trends of RES curtailment rate when the penalty and contract price are variable. The reference value of contract price and renege penalty here are 1 and 6 yuan/KWh, respectively. Total contract energy of unit 2 is $108 \mathrm{GWh}$ in the month. The surface is similar to curved fan-shape, where positive correlation was found between curtailment and penalty price with a negative one between curtailment and contract price.

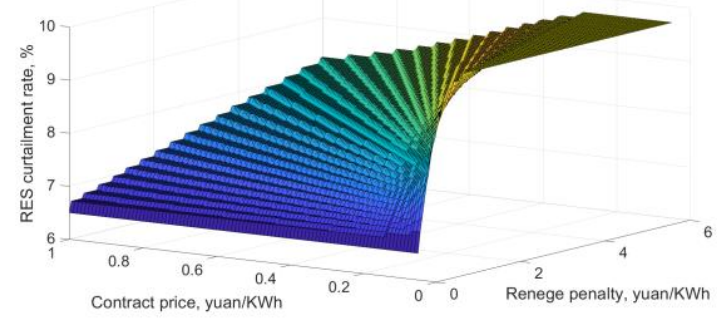

Fig. 5 Change of RES curtailment rate with renege penalty and contract price (contract energy is fixed in $108 \mathrm{GWh}$ )

The results of relationship between curtailment rate and penalty price under different contract energy is shown in Fig. 6 when the contract price is kept on 0.4 yuan/ KWh. When the contract energy is $86.4 \mathrm{GWh}$, meaning the minimum load capacity of unit 2 will cover the contract demand, there is no effect on original curtailment. The extreme scenario will happen when contract energy is $216 \mathrm{GWh}$, maximum generation energy in a month of unit 2, with penalty price of 6 yuan $/ \mathrm{KWh}$, leading to more than $30 \%$ RES curtailed.

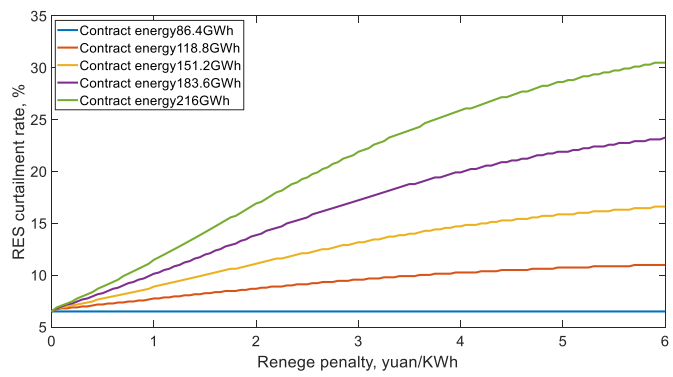

Fig. 6 Change of curtailment rate with penalty price under different contract energy

\subsection{Effect of physical contract}

The assumption in this case is only thermal unit 2 has a physical contract with a fixed execution load curve but no financial contract, which must be dispatched in operation. And the curve is the same in every day. We applied four typical shapes of contract curve in the case, displayed in Fig.7 (a). All the typical curves correspond to the same contract energy of $106 \mathrm{GWh}$ in one month. The curve of mode A has a similar tendency to system load with double peak-valley shape, while mode B is with reversed double peak-valley shape, mode $\mathrm{C}$ with fixed value and mode D with single peak-valley shape. The notable impact on the ELDC is found that mode A mainly decreases the rear tail of ELDC and mode $B$ reduce the former tail, shown in Fig.7 (b).
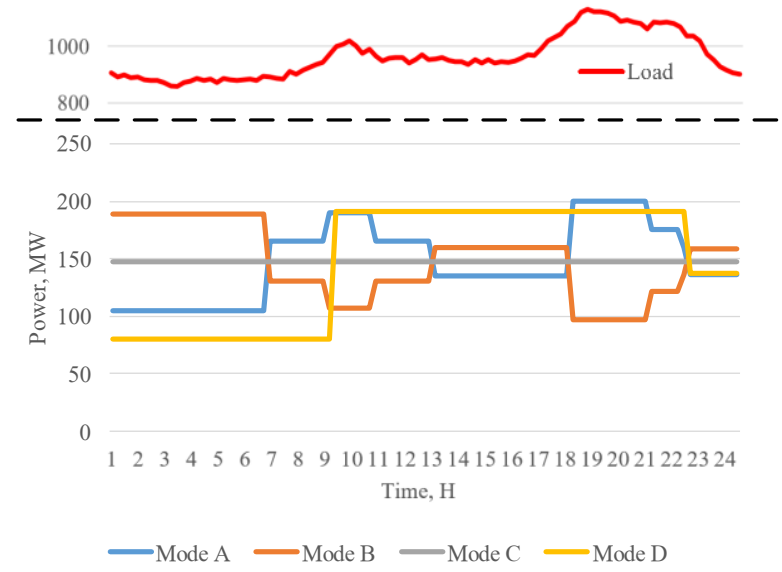

(a)Typical execution curve and system load

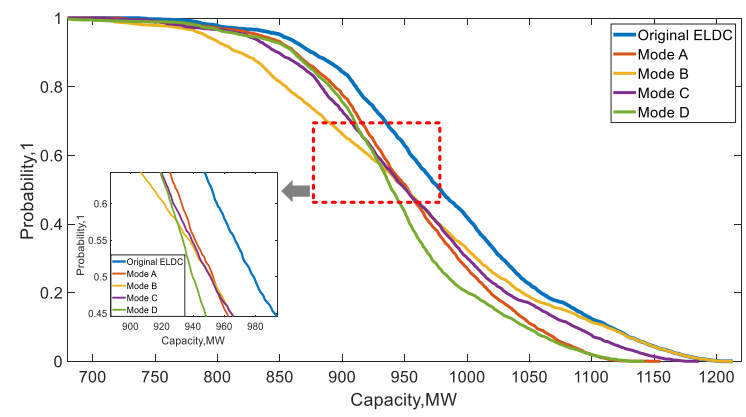

(b)The impact of physical contract on ELDC

Fig. 7 Typical execution curve of physical contract and impact on ELDC

The curtailment rate and energy with different modes are listed in Table 2. Compared to Fig. 5, we can see with the same total contract energy, physical contract has more severe impact. But the impact of mode $\mathrm{A}$ is the lightest and mode B the worst. It is apparent from this case that the curve shape impact curtailment a lot, and the shape in anti-load tendency is the worst choice.

Table 2 Curtailment rate and energy with four curve modes

\begin{tabular}{llllll}
\hline & Oringinal & $\begin{array}{l}\text { Mode } \\
\text { A }\end{array}$ & Mode B & Mode C & $\begin{array}{l}\text { Mode } \\
\text { D }\end{array}$ \\
\hline $\begin{array}{l}\text { Curtailment } \\
\text { rate/\% }\end{array}$ & 6.05 & $\mathbf{8 . 2 6}$ & 10.83 & 8.93 & 9.29 \\
$\begin{array}{l}\text { Curtailment } \\
\text { energy/MWh }\end{array}$ & 22852.6 & $\mathbf{2 9 0 1 4 . 4}$ & 38043.7 & 31387.4 & 32645.1 \\
\hline
\end{tabular}


A further trial is conducted in a larger data range where the curves in Fig.7 (a) are chosen as the reference and proportionally change the contract electricity from $50 \%$ to $150 \%$. The curtailment rate with four modes under different ratios is shown in Fig. 8. It can be found that the curtailment with mode $\mathrm{A}$ is the slightest when the ratio is between $90 \%$ and $150 \%$. Meanwhile, the result approves mode B is the worst contract mode whatever the ratio is.

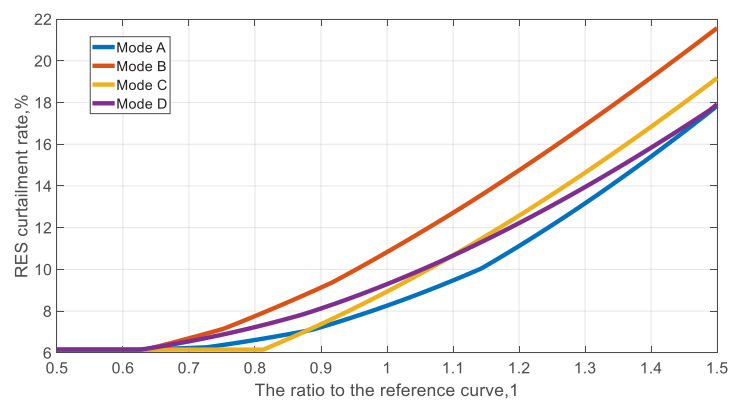

Fig. 8 RES curtailment rate with four types of curve in different ratio (compared to reference curve in Fig.7 (a))

\section{Conclusion}

This study set out to research the impact of forward market on system RES curtailment. The main types of forward market we studied are financial contract and physical contract where the stakeholders are thermal units and load entities. The evaluation model was built based on PPS, considering the probabilistic characteristic of forward contract, to calculate the RES curtailment rate in a month under different contract conditions. The experiments confirmed that forward contract of thermal unit will take up the generation share of RES. And physical contract has more impact than financial contract with the same contract electricity. The second major finding was that the impact of financial contract is sensitive to renege penalty and contract price, and physical contract sensitive to execution load curve. Taken together, these results suggest that the execution mode, price limitation and electricity decomposition curve of forward market must be considered carefully with the rapid increase of RES share. The study contributes to our understanding of relationship between forward market and RES accommodation. Further studies need to be carried out in order to research the influence of load entity price-response characteristics on the RES curtailment under forward market.

This work was supported by the State Grid Gansu Provincial
Electric Power Company Key Project (SGGSKY00FJWT1900170).

\section{References}

1. K. Ruddell, A. Downward, A. Philpott. Market power and forward prices, Economics Letters, 166: 6-9( 2018).

2. S. Li, S. Zhang, P. Andrews-Speed, Using diverse market-based approaches to integrate renewable energy: Experiences from China, Energy Policy, 125: 330-337( 2019).

3. $\mathrm{Xu} \mathrm{R}$, Song Z, Tang Q, Yu Z., The cost and marketability of renewable energy after power market reform in China: A review, Journal of Cleaner Production, 204: 409-424(2018).

4. K. C. Álvarez-Uribe, S. Arango-Aramburo, E. R. Larsen, Forward contracts in electricity markets and capacity investment: A simulation study, Utilities Policy, 54: 1-10(2018).

5. M. Banaei, M. Oloomi Buygi, H. Raouf Sheybani, Supply function Nash equilibrium of joint day-ahead electricity markets and forward contracts, International Journal of Electrical Power \& Energy Systems, 113: 104-116(2019).

6. P. C. Bhagwat, A. Marcheselli, J. C. Richstein, E. J.L. Chappin, L. J. De Vries, An analysis of a forward capacity market with long-term contracts, Energy Policy, 2017, 111: 255-267.

7. Ritz, Robert A . How does renewables competition affect forward contracting in electricity markets. Economics Letters, 146: 135-139(2016).

8. Miao S, Luo B, Shen J, et al. Short-term multiobjective hydro-thermal generation dispatch considering electricity market transition and mid-and long-term contract decomposition. Power System Technology, 42 (7) : 2221-2231(2018).

9. Guo Y, Kuang H, Guo S, et al. Research on weekly unit commitment strategy harmonizing long term market trade planning and daily generation scheduling, Power System Protection and Control, 45 (22) : 78-82(2017).

10. J. Wang, X. Wang and X. Ding, The Forward Contract Model of Interruptible Load in Power Market, IEEE/PES Transmission \& Distribution Conference \& Exposition: Asia and Pacific, Dalian, $1-5(2005)$. 